\title{
Self-dual vortices in Abelian Higgs models with dielectric function on the noncommutative plane
}

\author{
W. García Fuertes ${ }^{1, a}$, J. Mateos Guilarte ${ }^{2, b}$ \\ ${ }^{1}$ Departamento de Física, Facultad de Ciencias, Universidad de Oviedo, 33007 Oviedo, Spain \\ 2 Departamento de Física Fundamental and IUFFyM, Universidad de Salamanca, 37008 Salamanca, Spain
}

Received: 9 May 2014 / Accepted: 23 July 2014 / Published online: 21 August 2014

(C) The Author(s) 2014. This article is published with open access at Springerlink.com

\begin{abstract}
We show that Abelian Higgs Models with a dielectric function defined on the noncommutative plane enjoy self-dual vorticial solutions. By choosing a particular form of the dielectric function, we provide a family of solutions whose Higgs and magnetic fields interpolate between the profiles of the noncommutative Nielsen-Olesen and Chern-Simons vortices. This is done both for the usual $U(1)$ model and for the $S U(2) \times U(1)$ semilocal model with a doublet of complex scalar fields. The variety of known noncommutative self-dual vortices which display a regular behavior when the noncommutativity parameter tends to zero results in this way considerably enlarged.
\end{abstract}

\section{Introduction}

Although local quantum field theory has had an impressive success as a framework for describing the dynamics of elementary particles at the current accessible energies, there are indications that, at some stage in the route toward a more fundamental theory, the idea of locality as a basic assumption of physics should be given up. The exact way in which nonlocality would arise in that underlying theory is not clear, but a possibility that has often been considered by theorists is that, for lengths below some scale $\sqrt{\theta}$, spacetime has to be replaced by a different, blurred entity, in which the coordinates $x^{\mu}$ become noncommuting quantities $\hat{x}^{\mu}$ with commutators among them of order $\theta$. The reasons for considering noncommutative quantum field theories formulated on this arena are diverse. Originally, noncommutative QFT's appeared in an attempt to use the scale $\sqrt{\theta}$ as a cutoff for ultraviolet divergences, but later they were seen as effective theories on the spacetime foam resulting from the modified uncertainty principle arising in quantum gravity, as some

\footnotetext{
a e-mail: wifredo@uniovi.es

be-mail: guilarte@usal.es
}

low-energy limits of the theory of open strings propagating on a constant Kalb-Ramond field or as describing the low-energy quantum fluctuations of stacks of $D$-branes in the context of the IIB matrix model. The noncommutativity of spatial coordinates emerges also in condensed matter contexts, such as the motion of very light charged particles in strong magnetic fields as happens in the quantum Hall effect. For reviews of the formalism of noncommutative quantum field theory and some of its motivations and uses or their possible role in phenomenology; see [1-4].

The study of the different classes of solitons appearing in field and string theory is an important topic, both because they are stable objects with interesting dynamical behavior and because their conserved charges allow an interpretation of the solitons as supersymmetric BPS states, which can give significant information on the nonperturbative regime of the theory. In this respect, noncommutative QFT are especially appealing, because they can accomodate regular solitonic solutions in situations in which the usual commutative field theory would give singularities. This happens because Derrick's theorem, which is based on the scaling properties of the lagrangian kinetic- and potential-energy terms under dilatations of the coordinates, ceases to be valid in the noncommutative case due to the presence of the fundamental length $\sqrt{\theta}$. As a consequence, it is possible to find noncommutative scalar solitons even in theories without kinetic terms, and there is even a so-called solution generating technique which can be used to construct scalar and gauge solitons starting from trivial vacuum solutions [5]. This kind of solitons, however, become singular when the noncommutativity parameter $\theta$ is driven to zero.

In this paper we are going to study the self-dual vortices arising in a class of noncommutative Abelian models in which the kinetic Maxwell term incorporates a dielectric factor which is a function of the Higgs field. This dielectric contribution to the action, which spoils renormalizability, is however, a common occurrence in the effective truncation to low 
energy of supersymmetric theories. In the commutative case, self-dual vortices in Abelian models with dielectric function have been studied in [6-8] or [9], and other related Higgs models which arise from effective supersymmetric theories are dealt with in $[10,11]$ and [12]. Here, moving to the noncommutative plane, we will consider two variants among this kind of Abelian systems. First, we will pay attention to the case where there is only one complex scalar field and the local symmetry group is $U(1)$; this is the simplest paradigm for the Higgs mechanism and, from the phenomenological side, has interest as a Ginzburg-Landau model for superconductivity (the scalar field is the order parameter between type I and II superconductivities). Then we will extend the treatment to consider a model with a doublet of scalar fields enjoying a mixture of global $S U(2)$ and local $U(1)$ symmetries; this semilocal situation is a quite interesting limit of the electroweak theory and has been a subject of research in the field of cosmic strings. In both cases, we will focus on self-dual solutions which continue to be regular when $\theta$ goes to zero. For that, we follow closely the treatment given in the articles [13] and [14] by Lozano, Moreno and Schaposnik. In these references, the authors solve the self-duality equations for, respectively, noncommutative Nielsen-Olesen and Chern-Simons-Higgs $U(1)$ vortices by means of a very convenient ansatz which leads to some discrete recurrence relations. On the other hand, in [7] a specific form of the dielectric function which interpolates between the commutative Nielsen-Olesen and Chern-Simons energy densities was proposed. We use this function (with a slightly different parametrization) to find the noncommutative vortices interpolating between those found in [13] and [14], and also between their semilocal counterparts. The main theme of this paper is thus to combine the flexibility provided by a dielectric function with the techniques to deal with the noncommutative self-dual equations developed by the authors of $[13,14]$ to show how the spectrum of self-dual noncommutative vortices with good behavior for $\theta \rightarrow 0$ can be considerably enlarged.

\section{The Abelian Higgs model with dielectric function and its self-duality equations}

We are working on a three-dimensional spacetime with coordinates $\left(x^{0}, x^{1}, x^{2}\right)$ and metric $\eta_{\mu \nu}=\operatorname{diag}(1,-1,-1)$, but the spatial coordinates $x^{1}, x^{2}$ are not real numbers but fuzzy variables with uncertainty relation

$\Delta x^{1} \Delta x^{2} \geq \frac{\theta}{2}$

where $\theta$ is some positive real number. In this setup, we shall consider a dynamical model containing a complex scalar field $\phi$ and a gauge field $A_{\mu}$ interacting through the action

$$
\begin{aligned}
S= & \int \mathrm{d}^{3} x\left\{-\frac{1}{4} G * F_{\mu \nu} * G * F^{\mu \nu}+D_{\mu} \phi * \overline{D^{\mu} \phi}\right. \\
& \left.-\frac{1}{2} W * W\right\},
\end{aligned}
$$

where the star stands for the Groenewold-Moyal product

$f(x) * g(x)=\left.\exp \left[\frac{i}{2} \theta^{i j} \frac{\partial}{\partial x^{i}} \frac{\partial}{\partial x^{j^{\prime}}}\right] f(x) g\left(x^{\prime}\right)\right|_{x=x^{\prime}}$.

The formalism of noncommutative gauge field theories is explained, for instance, in [15] or [16]. In this particular model, the scalar field transforms with the fundamental representation of the $U_{*}(1)$ gauge group:

$\phi \longrightarrow \Lambda * \phi \quad \bar{\phi} \longrightarrow \bar{\phi} * \Lambda^{\dagger}$,

while $A_{\mu}$ is a $U_{*}(1)$ connection

$A_{\mu} \longrightarrow \Lambda * A_{\mu} * \Lambda^{\dagger}+\frac{i}{e} \Lambda * \partial_{\mu} \Lambda^{\dagger}$,

such that the covariant derivative of the scalar field and the gauge field strength are

$$
\begin{aligned}
& D_{\mu} \phi=\partial_{\mu} \phi-i e A_{\mu} * \phi \\
& F_{\mu \nu}=\partial_{\mu} A_{\nu}-\partial_{\nu} A_{\mu}-i e\left(A_{\mu} * A_{\nu}-A_{\nu} * A_{\mu}\right) .
\end{aligned}
$$

The field $\phi$ is self-interacting through a potential quadratic in $W$, a function of the star product of $\phi$ and $\bar{\phi}, W=W(\phi * \bar{\phi})$. Also, we allow for a non-minimal scalar-gauge interaction driven by the dielectric function $G=G(\phi * \bar{\phi})$. In this way, $G$ and $W$ transforms under the adjoint representation of the gauge group

$G \longrightarrow \Lambda * G * \Lambda^{\dagger} \quad W \longrightarrow \Lambda * W * \Lambda^{\dagger}$

exactly as $F_{\mu \nu}$ does, so that the gauge invariance of the action is guaranteed. In the following, we will also assume that $G$ is positive definite and that $W$ vanishes only when the product $\phi * \bar{\phi}$ takes its vacuum expectation value, denoted $v^{2}$.

Going to the temporal gauge $A_{0}=0$ and after some convenient rescalings

$A_{\mu} \rightarrow \frac{1}{e} A_{\mu} \quad \phi \rightarrow \frac{1}{e} \phi \quad v \rightarrow \frac{1}{e} v$,

the energy $E$ of the static field configurations takes the form

$$
\begin{aligned}
e^{2} E= & \int \mathrm{d}^{2} x\left\{\frac{1}{2} G * B * G * B+D_{k} \phi * \overline{D_{k} \phi}\right. \\
& \left.+\frac{1}{2} W * W\right\}
\end{aligned}
$$

where $B$ is the magnetic field

$B=F_{12}=\partial_{1} A_{2}-\partial_{2} A_{2}-i\left(A_{1} * A_{2}-A_{2} * A_{1}\right)$

and the spatial covariant derivatives are now $D_{k} \phi=\partial_{k} \phi-$ $i A_{k} * \phi, k=1,2$. This form of the energy functional is 
amenable to a Bogomolny splitting. The quadratic term in the covariant derivatives of the Higgs field is written as [17]

$$
\begin{aligned}
& \int \mathrm{d}^{2} x D_{k} \phi * \overline{D_{k} \phi} \\
& =\int \mathrm{d}^{2} x\left\{\left(D_{1} \phi+i D_{2} \phi\right) *\left(\overline{D_{1} \phi}-i \overline{D_{2} \phi}\right)+\phi * \bar{\phi} * B\right\}
\end{aligned}
$$

where an irrelevant contour term has been discarded, and the other two terms can be arranged as

$$
\begin{aligned}
& \int \mathrm{d}^{2} x\left\{\frac{1}{2} G * B * G * B+\frac{1}{2} W * W\right\} \\
& =\int \mathrm{d}^{2} x\left\{\frac{1}{2}(G * B+W)^{2}-W * G * B\right\},
\end{aligned}
$$

where the square is in the sense of the $*$-operation and the cyclic property

$\int \mathrm{d}^{2} x f(x) * g(x) * h(x)=\int \mathrm{d}^{2} x h(x) * f(x) * g(x)$

of the Groenewold-Moyal product has been used. By combining (3) and (4), we see that, if $W$ is chosen in such a way that

$W * G=\phi * \bar{\phi}-v^{2}$,

the energy of the field configurations which satisfy the selfduality equations

$$
\begin{aligned}
& G * B=-W, \\
& D_{1} \phi+i D_{2} \phi=0
\end{aligned}
$$

is proportional to the magnetic flux

$$
e^{2} E=v^{2} \int \mathrm{d}^{2} x B,
$$

which is indeed a boundary term by virtue of

$$
\int \mathrm{d}^{2} x A_{1}(x) * A_{2}(x)=\int \mathrm{d}^{2} x A_{2}(x) * A_{1}(x) .
$$

For finite-energy configurations, the fields at infinity depend only on the polar angle. The derivatives entering in (2) are therefore proportional to inverse powers of distance and then, in the asymptotic region of the noncommutative plane, the star product of fields converges to the ordinary product. This means that the classification in topological sectors can be directly taken over from the well-known results valid in the commutative plane. In particular, the magnetic flux is quantized. Hence, the solutions of (6)-(7) minimize the energy in each topological sector and are, therefore, bona fide solutions of the Euler-Lagrange equations. If we now denote by $\frac{1}{G}$ the inverse of $G$ according to the star product and take into account that $W$ and $G$ commute between themselves because both are functions of $\phi * \bar{\phi}$, the use of the constraint (5) turns the first self-duality equation into the more convenient form

$B=\left(\frac{1}{G}\right)^{2} *\left(v^{2}-\phi * \bar{\phi}\right)$,

to be used in what follows.

Functions on the noncommutative plane can be traded by operators on the Hilbert space $\mathcal{H}=L^{2}\left(\mathbf{R}^{2}\right)$ by means of the Weyl map

$f\left(x^{1}, x^{2}\right) \longrightarrow \hat{O}_{f}\left(\hat{x}^{1}, \hat{x}^{2}\right)=\frac{1}{(2 \pi)^{2}} \int \mathrm{d}^{2} k \hat{\Delta}(k) \tilde{f}(k)$,

where the Weyl kernel is $\hat{\Delta}(k)=\exp \left[-i\left(k_{1} \hat{x}_{1}+k_{2} \hat{x}_{2}\right)\right]$ and $\tilde{f}(k)=\int \mathrm{d}^{2} x e^{i k \cdot x} f(x)$ is the Fourier transform of $f(x)$; see $[5,15]$. The transformation is consistent in the sense that the star products are mapped to ordinary operator products on the Hilbert space. The use of the operator side of the Weyl map is very convenient for dealing with the self-duality equations, especially if we express them in holomorphic coordinates

$z=\frac{x^{1}+i x^{2}}{\sqrt{2}} \quad \bar{z}=\frac{x^{1}-i x^{2}}{\sqrt{2}}$

and introduce the harmonic oscillator ladder operators

$\hat{a}=\frac{\hat{x}^{1}+i \hat{x}^{2}}{\sqrt{2 \theta}} \quad \hat{a}^{\dagger}=\frac{\hat{x}^{1}+i \hat{x}^{2}}{\sqrt{2 \theta}}$

with commutator $\left[\hat{a}, \hat{a}^{\dagger}\right]=1$ consistent with the uncertainty relation (1). One can check $[13,14]$ that, in terms of these operators, the self-duality equations have the form

$$
\begin{aligned}
& -\frac{1}{\sqrt{\theta}}\left[a^{\dagger}, A_{\bar{z}}\right]-\frac{1}{\sqrt{\theta}}\left[a, A_{z}\right]-i\left[A_{z}, A_{\bar{z}}\right] \\
& =\left(\frac{i}{G}\right)^{2}\left(v^{2}-\phi \bar{\phi}\right), \\
& \frac{1}{\sqrt{\theta}}[a, \phi]-i A_{\bar{z}} \phi=0
\end{aligned}
$$

with $\phi, A_{z}$ and $A_{\bar{z}}$ representing here the operators $\hat{O}_{\phi}, \hat{O}_{A_{z}}$ and $\hat{O}_{A_{\bar{z}}}$ arising by applying the Weyl map to the Higgs and gauge fields of the original theory, but all hats have been suppressed to alleviate notational cluttering. Also, the vortex energy can be now computed as the trace

$e^{2} E=2 \pi \theta v^{2} \operatorname{Tr}_{\mathcal{H}} B$

on the Hilbert space.

\section{The interpolating model: noncommutative vortices}

By choosing the dielectric function in different forms it is possible to find self-dual noncommutative vortices with gauge and scalar fields displaying a wide variety of profiles. In particular, an interesting option proposed in [7] is to fix 
$G(\phi * \bar{\phi})$ in such a way that it can accommodate the profiles of the two most prominent types of vortices from a physical point of view: the Nielsen-Olesen and Chern-Simons vortices. This can be achieved by using

$G=\frac{1}{\sqrt{(1-\lambda)+\lambda \beta \phi * \bar{\phi}}}$

where the square root should be understood in the sense of the star product, $\lambda$ is a non-dimensional parameter with values in the interval $[0,1]$ and $\beta$ is an arbitrary constant with inverse mass squared dimension. Thus, the self-dual equations for this model are

$$
\begin{aligned}
& -\frac{1}{\sqrt{\theta}}\left[a^{\dagger}, A_{\bar{z}}\right]-\frac{1}{\sqrt{\theta}}\left[a, A_{z}\right]-i\left[A_{z}, A_{\bar{z}}\right] \\
& \quad=i[(1-\lambda)+\lambda \beta \phi \bar{\phi}]\left(v^{2}-\phi \bar{\phi}\right), \\
& \frac{1}{\sqrt{\theta}}[a, \phi]-i A_{\bar{z}} \phi=0 .
\end{aligned}
$$

For $\lambda=0$, these equations are precisely the self-dual equations of the ordinary Abelian Higgs Model [13], while for $\lambda=1$ they coincide with those of the relativistic ChernSimons-Higgs model [14], with the Chern-Simons $\kappa$ coupling given by $\kappa^{2}=\frac{1}{2 \beta}$. Thus, by continuously varying $\lambda$ between 0 and 1 we can find vortices with field profiles which interpolate between the solutions arising in these two theories.

\subsection{Solving the noncommutative vortex equation}

Let us first consider, following [17] where more details can be found, the case of very large noncommutative parameter $\theta$. By expanding in inverse powers of $\theta$

$\phi=\phi_{\infty}+\frac{1}{\theta} \phi_{-1}+\cdots$

$A_{\bar{z}}=\frac{1}{\sqrt{\theta}}\left(\left(A_{\bar{z}}\right)_{\infty}+\frac{1}{\theta}\left(A_{\bar{z}}\right)_{-1}+\cdots\right)$

the self-dual equations for general $\lambda$ are, to leading order, exactly the same that arise for Nielsen-Olesen vortices:

$\phi_{\infty} \bar{\phi}_{\infty}=v^{2}$

$i\left(A_{\bar{z}}\right)_{\infty}=\left[a, \phi_{\infty}\right]$.

As is well known [17,18], these equations have a solution for each positive integer $n$ which can be expressed in terms of the shift operators $|k\rangle\langle k+n|$ for the harmonic oscillator:

$$
\begin{aligned}
& \phi_{\infty}=v \sum_{k=0}^{\infty}|k\rangle\langle k+n|, \\
& \left(A_{\bar{z}}\right)_{\infty}=i \sum_{k=0}^{\infty}(\sqrt{k+1+n}-\sqrt{k+1})|k\rangle\langle k+1|
\end{aligned}
$$

Because

$a^{n}|k+n\rangle=\sqrt{(k+n)(k+n-1) \cdots(k+1)}|k\rangle$,

the scalar field operator can be recast as

$\phi_{\infty}=\frac{v}{\sqrt{a^{n}\left(a^{\dagger}\right)^{n}}} a^{n}$

and, in this way, the vorticial character of the solution is apparent through the factor $a^{n}$ (which is the noncommutative guise of the familiar angular dependence of type $z^{n}$ for commutative vortices). This character can be corroborated by computing the magnetic field, which is proportional to the projector onto the $|0\rangle$ state,

$$
\begin{aligned}
B_{\infty}= & -i F_{z \bar{z}}=\frac{i}{\theta}\left[a^{\dagger},\left(A_{\bar{z}}\right)_{\infty}\right]+\frac{i}{\theta}\left[a,\left(A_{z}\right)_{\infty}\right] \\
& -\frac{1}{\theta}\left[\left(A_{z}\right)_{\infty},\left(A_{\bar{z})_{\infty}}\right]=\frac{n}{\theta}|0\rangle\langle 0|,\right.
\end{aligned}
$$

and thus checking that the solution contains $n$ quanta of the magnetic flux

$\Phi_{M}=2 \pi \theta \operatorname{Tr}_{\mathcal{H}} B_{\infty}=2 \pi n$

as is appropriate for a vortex.

However, the presence of $\theta$ in the denominator of the magnetic field shows that these solutions will become singular if we try to extend them to the commutative $\theta=0$ case. In order to obtain a solution valid for all values of $\theta$, it is natural to modify the solution (9)-(10) for the $\theta=\infty$ case by trying an ansatz with a different coefficient for each shift operator,

$$
\begin{aligned}
\phi & =v \sum_{k=0}^{\infty} f_{k}|k\rangle\langle k+n|, \\
A_{\bar{z}} & =-\frac{i}{\sqrt{\theta}} \sum_{k=0}^{\infty} d_{k}|k\rangle\langle k+1|,
\end{aligned}
$$

which was proposed for the Abelian Higgs Models in [13] and for the Chern-Simons-Higgs Model in [14]. By substitution in the self-dual equations, one finds a system of algebraic equations for the $f_{k}$ and $d_{k}$ coefficients,

$$
\begin{aligned}
& d_{k} f_{k+1}=\sqrt{k+1} f_{k+1}-\sqrt{k+n+1} f_{k} \\
& 2 \sqrt{k} d_{k-1}-2 \sqrt{k+1} d_{k}+d_{k}^{2}-d_{k-1}^{2} \\
& \quad=\theta v^{2}\left(1-\lambda+\lambda \beta v^{2} f_{k}^{2}\right)\left(1-f_{k}^{2}\right)
\end{aligned}
$$

which can be solved along the lines explained in these references. By writing $d_{k}$ as $d_{k}=\sqrt{k+1}-\sqrt{k+n+1}+e_{k}$ the first equation gives the new coefficient $e_{k}$ in terms of the $f_{j}$ coefficients as

$e_{k}=\sqrt{k+n+1}\left(1-\frac{f_{k}}{f_{k+1}}\right)$ 
and, with this expression for $e_{k}$, the second equation yields a three term recurrence relation for the $f_{k}$,

$$
\begin{aligned}
& f_{k+1}^{2}\left[(k+n) f_{k-1}^{2}+f_{k}^{2}\left(1+\theta v^{2}\left(1-\lambda+\lambda \beta v^{2} f_{k}^{2}\right)\right.\right. \\
& \left.\left.\quad \times\left(1-f_{k}^{2}\right)\right)\right]=(k+n+1) f_{k}^{4},
\end{aligned}
$$

which gives $f_{k+1}$ in terms of $f_{k}$ and $f_{k-1}$. As, on the other hand, $f_{-1}=0, f_{1}$ is only a function of $f_{0}$,

$f_{1}^{2}=\frac{(n+1) f_{0}^{2}}{1+\theta v^{2}\left(1-\lambda+\lambda \beta v^{2} f_{0}^{2}\right)\left(1-f_{0}^{2}\right)}$.

Thus, once $f_{0}$ is chosen and $f_{1}$ determined, all the remaining coefficients can be recursively found. The task is to find the value of $f_{0}$ which matches the boundary condition for $k \rightarrow$ $\infty$ : in this limit, the $f_{k}$ have to approach unity, which is the only fixed point of the recurrence relation, and to accomplish it a simple bisection method can be used: we try first with $f_{0}^{2}=0.5$; if we find that $f_{k}$ grows over unity before $f_{k}<$ $f_{k-1}, f_{0}$ is too large and we change $f_{0}$ to $f_{0}^{2}=0.25$; if, instead, $f_{k}<f_{k-1}$ before $f_{k}>1, f_{0}$ is too small and we try with $f_{0}^{2}=0.75$. We repeat this procedure until a good matching with the boundary condition is attained. Once $f_{0}$ and all the $f_{k}$ coefficients are known, the magnetic field can be calculated from the self-duality equations as

$B=v^{2} \sum_{k=0}^{\infty}\left[1-\lambda+\lambda \beta v^{2} f_{k}^{2}\right]\left(1-f_{k}^{2}\right)|k\rangle\langle k|$

and, thus, the magnetic flux and energy are

$$
\begin{aligned}
\Phi_{M} & =2 \pi \theta \operatorname{Tr}_{\mathcal{H}} B \\
& =2 \pi \theta v^{2} \sum_{k=0}^{\infty}\left[1-\lambda+\lambda \beta v^{2} f_{k}^{2}\right]\left(1-f_{k}^{2}\right)
\end{aligned}
$$

and

$E=\frac{v^{2}}{e^{2}} \Phi_{M}$

In fact, for topological reasons, we expect that $\Phi_{M}=2 \pi n$, irrespective of the values of $\theta, \lambda, \beta$ or $v$, for any solution of the self-duality equations.

We have computed the correct value of $f_{0}$ for several values of the parameters in the dielectric function and for topological number $n=1$. For the case $\lambda=0, \beta$ disappears form the action and only the non-dimensional combination $\theta v^{2}$ matters. The results are shown in Table 1 . For the other extreme case $\lambda=1$, the parameters merge in a global factor $\beta \theta v^{4}$ and the results appear in Table 2. The numbers in Tables 1 and 2 are in good agreement with those obtained in $[13,14]$. In the Tables $3,4,5$ and 6 , we present the results for $f_{0}^{2}$ for some values of $\lambda$ interpolating between the NielsenOlesen and Chern-Simons cases. In these tables, the rows and columns correspond, respectively, to the constant values of $\theta v^{2}$ and $\beta v^{2}$ given in the margins.
3.2 Comparison with the commutative vortices

For all cases shown in the previous subsection, we have checked using (14) that the magnetic flux takes the value $\Phi_{M}=2 \pi$, as it should. As was done in [13], it is also interesting to check if the vortices of the noncommutative model converge to those of the commutative one when the parameter $\theta$ goes to zero. Using the ansatz

$\phi=v g(r) e^{i n \varphi}$

$A_{\theta}=n-\alpha(r)$

with $r$ and $\varphi$ the standard polar coordinates, the self-duality equations of the commutative model are [6-8]

$$
\begin{aligned}
& \frac{1}{r} \frac{\mathrm{d} \alpha}{\mathrm{d} r}=\left(1-\lambda+\lambda \beta v^{2} g^{2}\right)\left(g^{2}-1\right) \\
& \frac{\mathrm{d} g}{\mathrm{~d} r}=\frac{\alpha g}{r},
\end{aligned}
$$

and the boundary conditions take the form

$$
\begin{array}{ll}
g(0)=0 & g(\infty)=1 \\
\alpha(0)=n & \alpha(\infty)=0 .
\end{array}
$$

For $r \simeq 0$, the solution is

$g(r) \simeq g_{0} r^{n}$

$\alpha(r) \simeq n+\frac{\lambda-1}{2} r^{2}$

and starting with this asymptotics, the equations can be integrated numerically to find the value of $g_{0}$ which matches the boundary conditions at infinity. We have done this with a fourth-order Runge-Kutta method for different values of $\lambda$ and $\beta v^{2}$ and found the results for $g_{0}^{2}$ appearing in Table 7. We have, on the other hand, computed $f_{0}^{2}$ for very small $\theta$ for the diverse values of $\beta v^{2}$ and $\lambda$ shown in the table and we find a perfect agreement between $g_{0}^{2}$ in the commutative model and $\frac{f_{0}^{2}}{2 \theta v^{2}}$ in the noncommutative one, exactly as was established for the case $G=1$ in [13]. To understand this coincidence, let us write the scalar field of the noncommutative $n=1$ vortex as

$\phi=\frac{v}{\sqrt{a a^{\dagger}}} f\left(a^{\dagger} a\right) a$ with $f\left(a^{\dagger} a\right)|k\rangle=f_{k}|k\rangle$

and compute the expected value on the coherent state $|w\rangle=$ $e^{-\frac{|w|^{2}}{2}} e^{w a^{\dagger}}|0\rangle$, which satisfies

$\left\langle w\left|x^{1}+i x^{2}\right| w\right\rangle=\sqrt{2 \theta}(\operatorname{Re} w+i \operatorname{Im} w)$

and represents a minimal wavepacket which is centered around the point $\sqrt{2 \theta} w$ of the noncommutative plane and has spread $\Delta x^{1}=\Delta x^{2}=\sqrt{\frac{\theta}{2}}$ [17]. Now, using

$$
\begin{aligned}
& \langle w|\phi| w\rangle \\
& =\left\langle 0\left|\frac{v}{\sqrt{(a+w)\left(a^{\dagger}+\bar{w}\right)}} f\left(\left(a^{\dagger}+\bar{w}\right)(a+w)\right)(a+w)\right| 0\right\rangle
\end{aligned}
$$


Table 1 Values of $f_{0}^{2}$ for the case $\lambda=0$

\begin{tabular}{lllllllll}
\hline$\theta v^{2}$ & 0.25 & 0.50 & 0.75 & 1.00 & 1.25 & 1.50 & 1.75 & 2.00 \\
$f_{0}^{2}$ & 0.2572165 & 0.4006888 & 0.4940118 & 0.5602955 & 0.6101472 & 0.6491837 & 0.6806831 & 0.7066985 \\
\hline
\end{tabular}

Table 2 Values of $f_{0}^{2}$ for the case $\lambda=1$

\begin{tabular}{lllllllll}
\hline$\beta \theta v^{4}$ & 0.25 & 0.50 & 0.75 & 1.00 & 1.25 & 1.50 & 1.75 & 2.00 \\
$f_{0}^{2}$ & 0.1082514 & 0.2168143 & 0.3170487 & 0.4037747 & 0.4758857 & 0.5348773 & 0.5831093 & 0.6228436 \\
\hline
\end{tabular}

Table 3 Values of $f_{0}^{2}$ for the case $\lambda=0.2$

\begin{tabular}{lllllllll}
\hline$\beta v^{2} \downarrow / \theta v^{2} \rightarrow$ & 0.25 & 0.50 & 0.75 & 1.00 & 1.25 & 1.50 & 1.75 & 2.00 \\
\hline 0.25 & 0.2225542 & 0.3578602 & 0.4500099 & 0.5173522 & 0.5689932 & 0.6100056 & 0.6434570 & 0.6713201 \\
0.50 & 0.2267165 & 0.3642712 & 0.4576113 & 0.5255624 & 0.5774821 & 0.6185802 & 0.6520019 & 0.6797658 \\
0.75 & 0.2308680 & 0.3706328 & 0.4651091 & 0.5336139 & 0.5857629 & 0.6269045 & 0.6602619 & 0.6878986 \\
1.00 & 0.2350088 & 0.3769440 & 0.4725019 & 0.5415063 & 0.5938368 & 0.6349824 & 0.6682435 & 0.6957276 \\
1.25 & 0.2391388 & 0.3832038 & 0.4797885 & 0.5492392 & 0.6017055 & 0.6428179 & 0.6759534 & 0.7032625 \\
1.50 & 0.2432580 & 0.3894111 & 0.4869678 & 0.5568126 & 0.6093711 & 0.6504157 & 0.6833991 & 0.7105131 \\
1.75 & 0.2473662 & 0.3955651 & 0.4940389 & 0.5642271 & 0.6168360 & 0.6577808 & 0.6905881 & 0.7174894 \\
2.00 & 0.2514633 & 0.4016646 & 0.5010010 & 0.5714831 & 0.6241032 & 0.6649185 & 0.6975281 & 0.7242017 \\
\hline
\end{tabular}

Table 4 Values of $f_{0}^{2}$ for the case $\lambda=0.4$

\begin{tabular}{lllllllll}
\hline$\beta v^{2} \downarrow / \theta v^{2} \rightarrow$ & 0.25 & 0.50 & 0.75 & 1.00 & 1.25 & 1.50 & 1.75 & 2.00 \\
\hline 0.25 & 0.1834747 & 0.3064902 & 0.3952554 & 0.4626214 & 0.5156652 & 0.5586206 & 0.5941838 & 0.6241573 \\
0.50 & 0.1923526 & 0.3208740 & 0.4129240 & 0.4821936 & 0.5362822 & 0.5797419 & 0.6154649 & 0.6453767 \\
0.75 & 0.2011923 & 0.3350540 & 0.4301225 & 0.5010010 & 0.5558540 & 0.5995703 & 0.6352428 & 0.6649185 \\
1.00 & 0.2099940 & 0.3490185 & 0.4468313 & 0.5190293 & 0.5743853 & 0.6181386 & 0.6535835 & 0.6828842 \\
1.25 & 0.2187571 & 0.3627553 & 0.4630337 & 0.5362727 & 0.5918931 & 0.6354940 & 0.6705672 & 0.6993862 \\
1.50 & 0.2274800 & 0.3762523 & 0.4787170 & 0.5527332 & 0.6084050 & 0.6516942 & 0.6862819 & 0.7145411 \\
1.75 & 0.2361604 & 0.3894982 & 0.4938726 & 0.5684202 & 0.6239571 & 0.6668039 & 0.7008194 & 0.7284646 \\
2.00 & 0.2447957 & 0.4024825 & 0.5084961 & 0.5833496 & 0.6385915 & 0.6808915 & 0.7142714 & 0.7412679 \\
\hline
\end{tabular}

Table 5 Values of $f_{0}^{2}$ for the case $\lambda=0.6$

\begin{tabular}{lllllllll}
\hline$\beta v^{2} \downarrow / \theta v^{2} \rightarrow$ & 0.25 & 0.50 & 0.75 & 1.00 & 1.25 & 1.50 & 1.75 & 2.00 \\
\hline 0.25 & 0.1389372 & 0.2432580 & 0.3244385 & 0.3894111 & 0.4426027 & 0.4869678 & 0.5245515 & 0.5568126 \\
0.50 & 0.1531621 & 0.2677365 & 0.3559161 & 0.4255004 & 0.4816218 & 0.5277492 & 0.5662879 & 0.5989462 \\
0.75 & 0.1673229 & 0.2917726 & 0.3862109 & 0.4594802 & 0.5175747 & 0.5645702 & 0.6032743 & 0.6356563 \\
1.00 & 0.1814214 & 0.3153038 & 0.4151959 & 0.4912258 & 0.5504218 & 0.5975425 & 0.6358143 & 0.6674566 \\
1.25 & 0.1954515 & 0.3382635 & 0.4427719 & 0.5206897 & 0.5802441 & 0.6269188 & 0.6643456 & 0.6949639 \\
1.50 & 0.2094023 & 0.3605889 & 0.4688750 & 0.5478960 & 0.6072109 & 0.6530306 & 0.6893532 & 0.7187975 \\
1.75 & 0.2232606 & 0.3822249 & 0.4934771 & 0.5729255 & 0.6315447 & 0.6762369 & 0.7113119 & 0.7395235 \\
2.00 & 0.2370120 & 0.4031266 & 0.5165834 & 0.5958987 & 0.6534912 & 0.6968901 & 0.7306554 & 0.7576340 \\
\hline
\end{tabular}


Table 6 Values of $f_{0}^{2}$ for the case $\lambda=0.8$

\begin{tabular}{lllllllll}
\hline$\beta v^{2} \downarrow / \theta v^{2} \rightarrow$ & 0.25 & 0.50 & 0.75 & 1.00 & 1.25 & 1.50 & 1.75 & 2.00 \\
\hline 0.25 & 0.0874802 & 0.1626094 & 0.2274800 & 0.2838134 & 0.3330198 & 0.3762523 & 0.4144539 & 0.4483972 \\
0.50 & 0.1077297 & 0.2001111 & 0.2788254 & 0.3457787 & 0.4028276 & 0.4516387 & 0.4936371 & 0.5300064 \\
0.75 & 0.1279574 & 0.2370120 & 0.3279805 & 0.4031266 & 0.4651461 & 0.5165834 & 0.5595923 & 0.5958986 \\
1.00 & 0.1481567 & 0.2730445 & 0.3743037 & 0.4550554 & 0.5194259 & 0.5711959 & 0.6133762 & 0.6482294 \\
1.25 & 0.1682918 & 0.3079312 & 0.4173346 & 0.5013053 & 0.5660076 & 0.6166414 & 0.6570262 & 0.6898471 \\
1.50 & 0.1883157 & 0.3414295 & 0.4568418 & 0.5420656 & 0.6057372 & 0.6544386 & 0.6926365 & 0.7232946 \\
1.75 & 0.2081774 & 0.3733515 & 0.4928022 & 0.5778005 & 0.6396228 & 0.6860415 & 0.7219792 & 0.7505531 \\
2.00 & 0.2278258 & 0.4035707 & 0.5253498 & 0.6090921 & 0.6686338 & 0.7126815 & 0.7464413 & 0.7730916 \\
\hline
\end{tabular}

Table 7 Values of $g_{0}^{2}$ for commutative vortices

\begin{tabular}{lllllllll}
\hline$\lambda \downarrow / \beta v^{2} \rightarrow$ & 0.25 & 0.50 & 0.75 & 1.00 & 1.25 & 1.50 & 1.75 & 2.00 \\
\hline 0.0 & 0.7279 & 0.7279 & 0.7279 & 0.7279 & 0.7279 & 0.7279 & 0.7279 & 0.7279 \\
0.2 & 0.5933 & 0.6042 & 0.6152 & 0.6260 & 0.6369 & 0.6478 & 0.6586 & 0.6695 \\
0.4 & 0.4586 & 0.4804 & 0.5021 & 0.5237 & 0.5453 & 0.5668 & 0.5882 & 0.6096 \\
0.6 & 0.3239 & 0.3563 & 0.3886 & 0.4207 & 0.4527 & 0.4846 & 0.5164 & 0.5482 \\
0.8 & 0.1889 & 0.2317 & 0.2741 & 0.3164 & 0.3586 & 0.4008 & 0.4429 & 0.4850 \\
1.0 & 0.0524 & 0.1049 & 0.1573 & 0.2098 & 0.2622 & 0.3147 & 0.3671 & 0.4195 \\
\hline
\end{tabular}

we see that for $w \rightarrow 0$ and in the limit $\theta \rightarrow 0$ we have

$\langle w|\phi| w\rangle \rightarrow v f_{0} w=\frac{v f_{0}}{\sqrt{2 \theta}}\left(x^{1}+i x^{2}\right)$

and this should be interpreted as the value of $\phi$ near the origin. On the other hand, for the commutative model $\phi \simeq g_{0} v^{2} r e^{\varphi}$, so one should expect $g_{0}^{2}=\frac{f_{0}^{2}}{2 \theta v^{2}}$, as indeed occurs.

\subsection{Noncommutative vortex profiles}

Once the scalar and magnetic field operators are known in Hilbert space, it is not difficult to invert the Weyl map and find the functional form of these vortex fields in the noncommutative coordinates. For that, we only have to take into account that the function $f_{j, k}(x)$ with the Weyl transform $|j\rangle\langle k|$ is [5]

$$
\begin{aligned}
& f_{j, k}(x) \\
& \quad=2(-1)^{j} \sqrt{\frac{j !}{k !}} e^{-\frac{r^{2}}{\theta}}\left(2 \frac{r^{2}}{\theta}\right)^{\frac{j-k}{k}} L_{j}^{k-j}\left(2 \frac{r^{2}}{\theta}\right) e^{i(k-j) \varphi}
\end{aligned}
$$

where $z=\frac{r}{\sqrt{2}} \exp i \varphi$ and the $L_{p}^{q}(y)$ are generalized Laguerre polynomials. In particular, as

$\phi \bar{\phi}=v^{2} \sum_{k=0}^{\infty} f_{k}^{2}|k\rangle\langle k|$

and the magnetic field is (14), one finds

$$
\begin{aligned}
& \phi(x) * \bar{\phi}(x)=v^{2} \sum_{k=0}^{\infty} f_{k}^{2} f_{k, k}(x) \\
& B(x)=v^{2} \sum_{k=0}^{\infty}\left(1-\lambda+\lambda \beta v^{2} f_{k}^{2}\right)\left(1-f_{k}^{2}\right) f_{k, k}(x) .
\end{aligned}
$$

Figures 1, 2, 3, 4, 5 and 6 show the profiles of $\phi * \bar{\phi}$ (in red) and $B$ (in green) as a function of $r$ for several values of the non-dimensional parameters $\theta v^{2}$ and $\beta v^{2}$. In each figure, the curves are for $\lambda=0,0.2,0.4,0.6,0.8$ and 1 , and one can distinguish among these values because in all cases both $\phi * \bar{\phi}$ and $B$ at the origin decrease with $\lambda$. A few comments about the figures:

- As one can see, the profile of the magnetic field exhibits a maximum at the center of the Nielsen-Olesen vortex, which is more and more flat as $\lambda$ increases, and becomes finally a minimum for the Chern-Simons case. Thus, the magnetic field concentrates at a peak for small $\lambda$ and is more disperse, forming a ring around the core of the vortex, as $\lambda$ approaches one. The effect is more noticeable when the parameter $\theta$ measuring the noncommutativity of the plane is small; in particular, in Fig. 4, which has the larger value of $\theta v^{2}$, the magnetic field at the center of $\lambda=1$ case looks more like a plateau than like a ring.

- The first four figures have $\beta v^{2}$ fixed and a increasing degree of noncommutativity, with parameter varying from $\theta v^{2}=0.25$ to $\theta v^{2}=1.5$. Looking at these, we see that, as $\theta v^{2}$ increases, the value of $B(0)$ decreases for the Nielsen- 


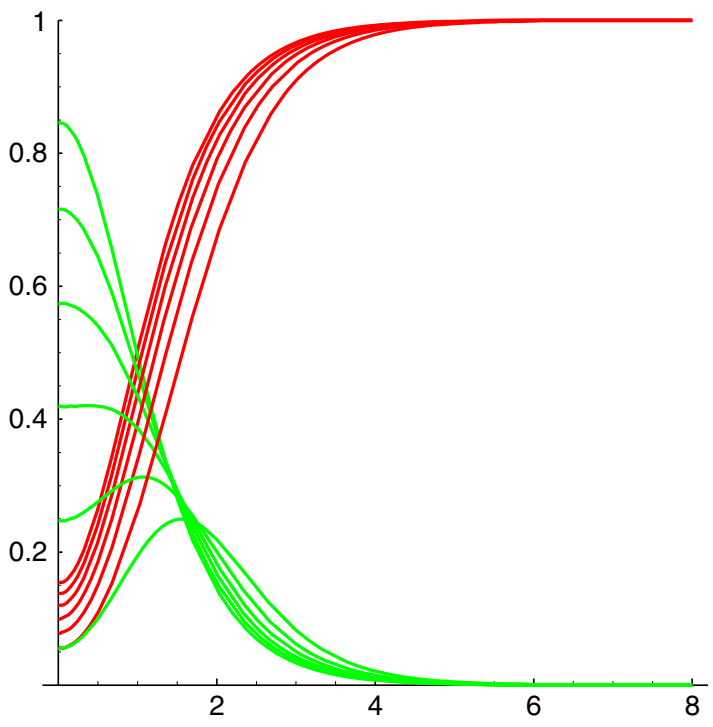

Fig. 1 The cases $\theta v^{2}=0.25, \beta v^{2}=1$

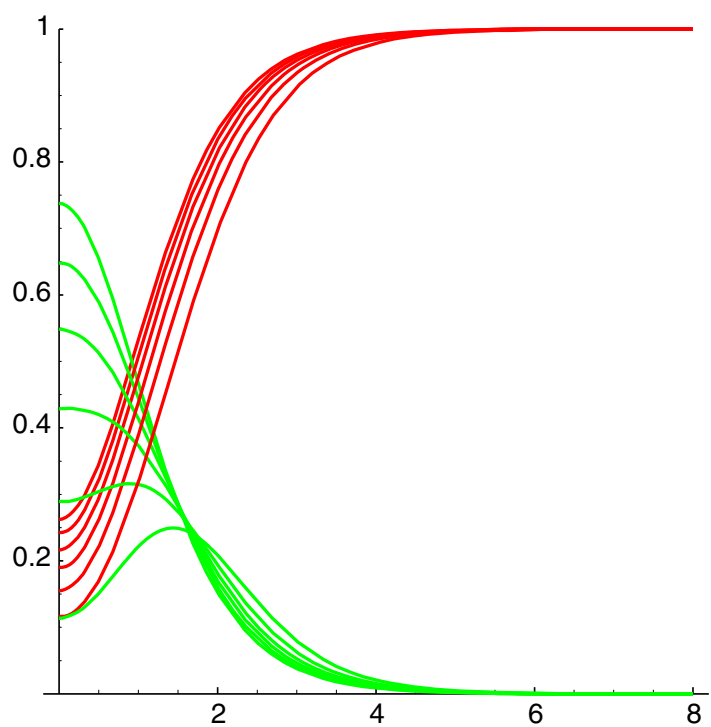

Fig. 2 The cases $\theta v^{2}=0.5, \beta v^{2}=1$

Olesen vortices, but increases for the Chern-Simons ones. Thus, while for small noncommutativity the magnetic field at the center of the vortex shows a wide variation with $\lambda$, for higher $\theta v^{2}$ the range of this variation is of lesser extent.

- In the same four figures, we can see that the profiles of $\phi * \bar{\phi}$ with distance are quite similar for all the values of $\lambda$, but $\phi(0) * \bar{\phi}(0)$ increases with $\theta v^{2}$. This is as it should be expected, given that for commutative vortices the scalar field vanishes at the origin.

- Figure 5 is for small noncommutativity and large $\beta v^{2}$, which corresponds to small Chern-Simons parameter $\kappa$. There is in this case a very important variation of the magnetic field with $\lambda$, and the ring-like shape of the core of the vortex is evident for quite low values of the interpolating

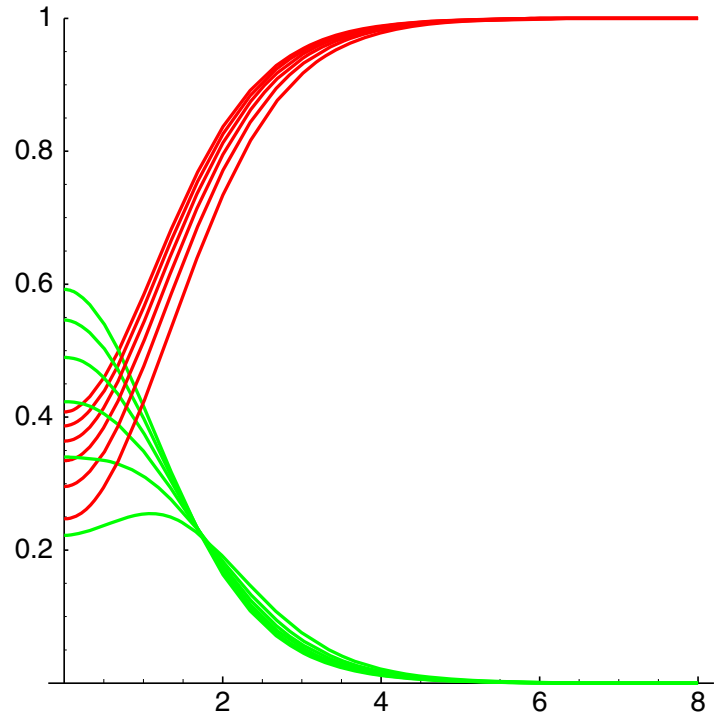

Fig. 3 The cases $\theta v^{2}=1, \beta v^{2}=1$

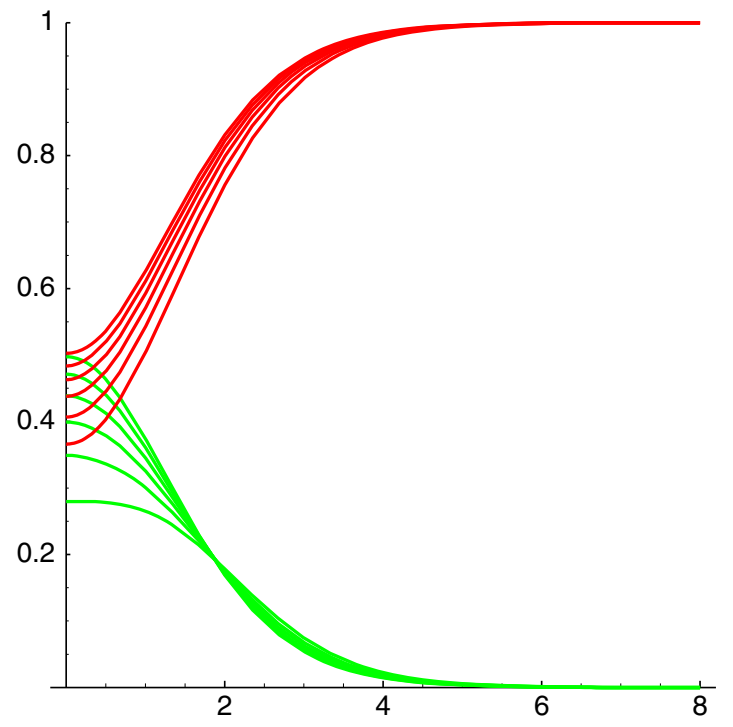

Fig. 4 The cases $\theta v^{2}=1.5, \beta v^{2}=1$

parameter. Instead, the dependence of the profile of $\phi * \bar{\phi}$ with $\lambda$ is completely negligible.

- That behavior is in contrast with Fig. 6, which has more amount of noncommutativity and a smaller $\beta v^{2}$. In this case, both the magnetic field $B$ and the scalar field magnitude $\phi * \bar{\phi}$ show substantial variations as we interpolate between the Nielsen-Olesen and Chern-Simons solutions.

\section{The semilocal model with dielectric function}

Unlike the AHM, the Standard Model of particle physics does not admit topologically stable vortices. The reason is 


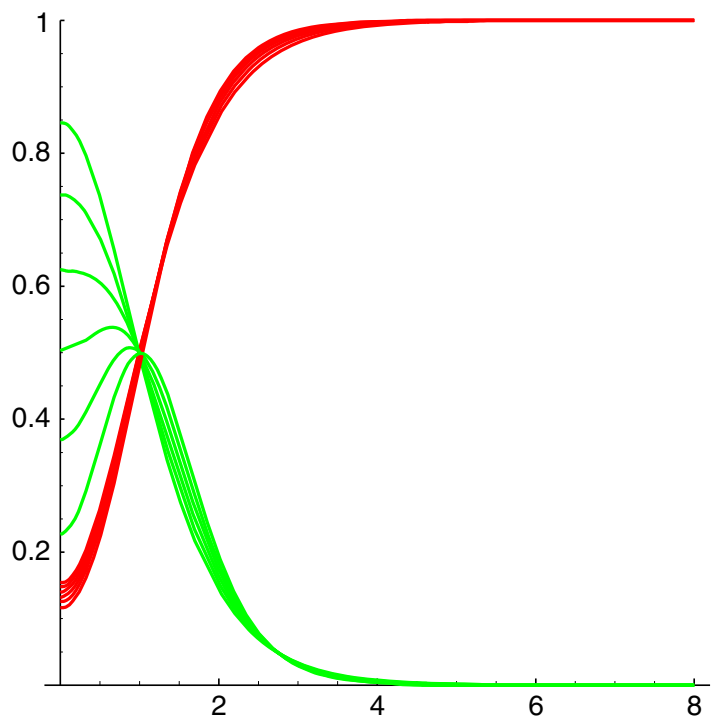

Fig. 5 The cases $\theta v^{2}=0.25, \beta v^{2}=2$

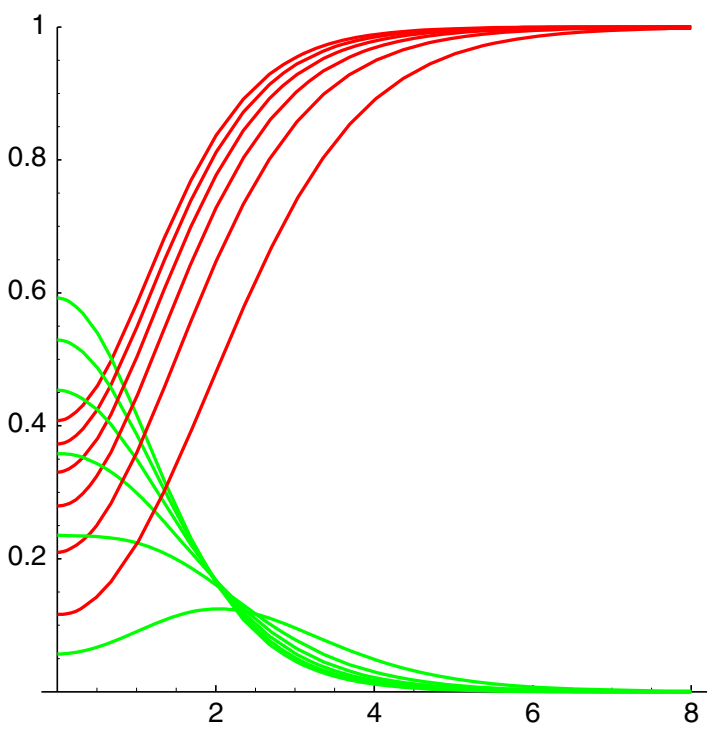

Fig. 6 The cases $\theta v^{2}=1, \beta v^{2}=0.5$

that, in this case, the pattern of gauge symmetry breaking is $G=S U(2) \times U(1) \rightarrow H=U(1)$ and the fundamental group of the quotient $G / H$ is trivial, $\pi_{1}(G / H)=1$. There is, however, an interesting exception to this general statement: if the Weinberg angle is $\theta_{W}=\frac{\pi}{2}$, the weak isospin gauge bosons decouple, the $S U(2)$ factor becomes a global symmetry and stable flux lines appear in the spectrum [19]. Being the consequence of the mixing of the global $S U(2)$ and gauge $U(1)$ symmetries, these solutions are known as semilocal vortices. Although the Higgs field is a $S U(2)$ doublet

$\Phi=\left(\begin{array}{c}\phi^{+} \\ \phi^{0}\end{array}\right)$ and thus the vacuum orbit is $S^{3}$, the stability of semilocal vortices is guaranteed because, to ensure the vanishing of their covariant derivatives at long distances, the asymptotic scalar field has to be given by a map from the spatial $S^{1}$ border to one $S^{1}$ fiber of the Hopf fibration $S^{3} \rightarrow S^{2}$ [20,21]. Hence, the effective fundamental group which classifies the finiteenergy configurations is $\pi_{1}\left(S^{1}\right)=\mathbf{Z}$, the winding number corresponding, as usual, to the magnetic flux. In each topological sector, the axially symmetric semilocal vortices form a family which is parametrized by a complex number and interpolates between standard Nielsen-Olesen vortices and $\mathbf{C} P^{1}$-lumps. Although all the defects in the family are stable [22], the fields decay exponentially at infinity only for the Nielsen-Olesen vortices. For the other cases the magnetic flux is more spread and the fields reach their asymptotic values as inverse powers of the distance.

\subsection{Semilocal self-dual noncommutative vortices}

Our aim in this section is to study the self-dual vortex solutions arising in a noncommutative semilocal model with dielectric function. With the rescalings seen in Sect. 2, the action of the model is

$$
\begin{aligned}
S= & \int \mathrm{d}^{3} x\left\{-\frac{1}{4} G * F_{\mu \nu} * G * F^{\mu \nu}\right. \\
& \left.+\sum_{a=+, 0} D_{\mu} \phi^{a} * \overline{D^{\mu} \phi^{a}}-\frac{1}{2} W * W\right\} .
\end{aligned}
$$

where $G$ and $W$ are positive functions which, to be covariant under both the global $S U(2)$ and the gauge $U(1)$ symmetries, have the structure:

$G=G\left(\sum_{a=0,+} \phi^{a} \bar{\phi}^{a}\right), \quad W=W\left(\sum_{a=0,+} \phi^{a} \bar{\phi}^{a}\right)$.

The energy for static configurations is

$$
\begin{aligned}
e^{2} E= & \int \mathrm{d}^{2} x\left\{\frac{1}{2} G * B * G * B\right. \\
& \left.+\sum_{a=0,+} D_{k} \phi^{a} * \overline{D_{k} \phi^{a}}+\frac{1}{2} W * W\right\}
\end{aligned}
$$

and, as in the previous section, one can perform a Bogomolny splitting such that if

$W * G=\sum_{a=+, 0} \phi^{a} * \bar{\phi}^{a}-v^{2}$,

the field configurations which satisfy the self-duality equations

$$
\begin{aligned}
& G * B=-W \\
& D_{1} \phi^{+}+i D_{2} \phi^{+}=0, \\
& D_{1} \phi^{0}+i D_{2} \phi^{0}=0
\end{aligned}
$$


saturate the Bogomolny bound

$e^{2} E \geq v^{2} \int \mathrm{d}^{2} x B$

As before, we choose a dielectric function

$$
G=\frac{1}{\sqrt{(1-\lambda)+\lambda \beta \sum_{a=+, 0} \phi^{a} * \bar{\phi}^{a}}},
$$

which is suitable to interpolate between semilocal vortices of Maxwell type and semilocal Chern-Simons vortices; for the latter, see [23]. ${ }^{1}$ Then the Bogomolny equations are

$$
\begin{aligned}
& -\frac{1}{\sqrt{\theta}}\left[a^{\dagger}, A_{\bar{z}}\right]-\frac{1}{\sqrt{\theta}}\left[a, A_{z}\right]-i\left[A_{z}, A_{\bar{z}}\right] \\
& =i\left[(1-\lambda)+\lambda \beta \sum_{a=+, 0} \phi^{a} \bar{\phi}^{a}\right]\left(v^{2}-\sum_{a=+, 0} \phi^{a} \bar{\phi}^{a}\right) \\
& \frac{1}{\sqrt{\theta}}\left[a, \phi^{+}\right]-i A_{\bar{z}} \phi^{+}=0, \\
& \frac{1}{\sqrt{\theta}}\left[a, \phi^{0}\right]-i A_{\bar{z}} \phi^{0}=0 .
\end{aligned}
$$

and a convenient ansatz to solve them in the sector of magnetic flux $\Phi_{M}=2 \pi n$ is a direct extension of (11)-(12):

$$
\begin{aligned}
\phi^{+} & =v \sum_{k=0}^{\infty} f_{k}|k\rangle\langle k+n|, \\
\phi^{0} & =v \sum_{k=0}^{\infty} \eta_{k}|k\rangle\langle k+l|, \\
A_{\bar{z}} & =-\frac{i}{\sqrt{\theta}} \sum_{k=0}^{\infty} d_{k}|k\rangle\langle k+1|,
\end{aligned}
$$

in which we have used the global $S U$ (2) symmetry to put the topological vorticity in the $\phi^{+}$component, i.e. we will use boundary conditions $f_{k} \rightarrow 1, \eta_{k} \rightarrow 0$ for $k \rightarrow \infty$, but we also allow for a behavior of type $a^{l}$ for the other component. This mimics the angular dependence of the solutions found for the commutative model, see [20,22], and the analysis of that case suggests that, in order to have well-behaved finiteenergy solutions, we have to take $0 \leq l \leq n-1$.

Using this ansatz in (16) and (17), we can relate the coefficients $\eta_{k}$ and $f_{k}$ through

$\eta_{k+1}=\sqrt{\frac{k+l+1}{k+n+1}} \frac{f_{k+1}}{f_{k}} \eta_{k}$

and then, iterating this relation and using the same arguments of the previous section, we see that, once some initial values

\footnotetext{
${ }^{1}$ Here we abide by the notation of [14]. To compare with [23], $A_{\mu}, \partial_{\mu}, \phi, \kappa$ and $\eta$ in that paper have to be rescaled according to $A_{\mu} \rightarrow \frac{A_{\mu}}{\eta}, x_{\mu} \rightarrow \eta x_{\mu}, \phi \rightarrow \frac{\sqrt{2} \phi}{\eta}, \kappa \rightarrow \frac{2 \kappa}{\eta}, \eta \rightarrow \sqrt{2} \eta$.
}

for $f_{0}$ and $\eta_{0}$ are given, all remaining coefficients follow from

$\eta_{k}=\sqrt{\frac{(k+l) ! n !}{(k+n) ! l !}} \frac{f_{k}}{f_{0}} \eta_{0}$,

the recurrence relation

$$
\begin{aligned}
(k+n+1) f_{k}^{4}= & {\left[(k+n) f_{k-1}^{2}+f_{k}^{2}\left(1+\theta v^{2}(1-\lambda\right.\right.} \\
& \left.\left.\left.+\lambda \beta v^{2}\left(f_{k}^{2}+\eta_{k}^{2}\right)\right)\left(1-f_{k}^{2}-\eta_{k}^{2}\right)\right)\right] f_{k+1}^{2},
\end{aligned}
$$

and (13). Thus, the problem is to find, for each $\eta_{0}$, the value of $f_{0}^{2}$ which gives the correct behavior for $k \rightarrow \infty$. Using the bisection method, we have found $f_{0}^{2}$ for $n=1, l=0$ and the cases given in the Tables 8, 9, 10, 11, 12, 13, 14 and 15.

\subsection{Comparison with the semilocal commutative vortices}

Let us now compare with the commutative semilocal model. With the radial ansatz

$\phi^{+}=v g(r) e^{i n \varphi}$,

$\phi^{0}=v h(r) e^{i l \varphi}$

$A_{\theta}=n-\alpha(r)$

the commutative Bogomolny equations are

$$
\begin{aligned}
& \frac{1 \mathrm{~d} \alpha}{r} \frac{\mathrm{d} r}{\mathrm{~d} g}=\left[1-\lambda+\lambda \beta v^{2}\left(g^{2}+h^{2}\right)\right]\left(g^{2}+h^{2}-1\right), \\
& \frac{\mathrm{d} g}{\mathrm{~d} r}=\frac{\alpha g}{r}, \\
& \frac{\mathrm{d} h}{\mathrm{~d} r}=\frac{\alpha-n+l}{r} h,
\end{aligned}
$$

to be solved with the boundary conditions

$g(0)=0 \quad h(0)=h_{0} \delta_{l, 0} \quad \alpha(0)=n$,

$g(\infty)=0 \quad h(\infty)=0 \quad \alpha(\infty)=0$.

Let us concentrate in the case $n=1, l=0$. From (22) and (23), it follows that $h(r)=\frac{\rho}{r} g(r)$ with $\rho=\frac{h_{0}}{g^{\prime}(0)}$. Using this in (21) one can see that the solution has the form

$g(r) \simeq g_{0} r$

$\alpha(r) \simeq 1+\frac{1}{2}\left(1+\lambda \beta v^{2}\left|h_{0}\right|^{2}-\lambda\right)\left(\left|h_{0}\right|^{2}-1\right) r^{2}$

when $r \simeq 0$. The integration of the equations by the RungeKutta method shows that the values of $g_{0}^{2}$ compatible with the boundary conditions at infinity are those appearing in Table 16 . We have checked that these are precisely the values of $\frac{f_{0}^{2}}{2 \theta v^{2}}$ obtained in the noncommutative model when we take the limit $\theta \rightarrow 0$, as they should. 
Table 8 Values of $f_{0}^{2}$ for the case $\lambda=0$

\begin{tabular}{lllllllll}
\hline$\eta_{0} \downarrow / \theta v^{2} \rightarrow$ & 0.25 & 0.50 & 0.75 & 1.00 & 1.25 & 1.50 & 1.75 & 2.00 \\
\hline 0.1 & 0.2542716 & 0.3962479 & 0.4886493 & 0.5543005 & 0.6036871 & 0.6423652 & 0.6735785 & 0.6993595 \\
0.2 & 0.2454721 & 0.3829659 & 0.4726012 & 0.5363519 & 0.5843404 & 0.6219402 & 0.6522926 & 0.6773681 \\
0.3 & 0.2309262 & 0.3609669 & 0.4459876 & 0.5065611 & 0.5522091 & 0.5880020 & 0.6169109 & 0.6408026 \\
0.4 & 0.2108201 & 0.3304652 & 0.4090162 & 0.4651210 & 0.5074699 & 0.5407120 & 0.5675811 & 0.5897986 \\
0.5 & 0.1854286 & 0.2917786 & 0.3619958 & 0.4123184 & 0.4503856 & 0.4803106 & 0.5045229 & 0.5245576 \\
0.6 & 0.1551343 & 0.2453514 & 0.3053590 & 0.3485556 & 0.3813253 & 0.4071348 & 0.4280443 & 0.4453617 \\
\hline
\end{tabular}

Table 9 Values of $f_{0}^{2}$ for the case $\lambda=1$

\begin{tabular}{lllllllll}
\hline$\eta_{0} \downarrow / \theta \beta v^{4} \rightarrow$ & 0.25 & 0.50 & 0.75 & 1.00 & 1.25 & 1.50 & 1.75 & 2.00 \\
\hline 0.1 & 0.1090283 & 0.2173627 & 0.3166714 & 0.4021973 & 0.4731451 & 0.5311410 & 0.5785618 & 0.6176438 \\
0.2 & 0.1109311 & 0.2183089 & 0.3147740 & 0.3967814 & 0.4643727 & 0.5195071 & 0.5645938 & 0.6017931 \\
0.3 & 0.1128118 & 0.2178051 & 0.3093577 & 0.3857451 & 0.4481253 & 0.4988503 & 0.5403345 & 0.5746132 \\
0.4 & 0.1131022 & 0.2134202 & 0.2978589 & 0.3668159 & 0.4225433 & 0.4676949 & 0.5046197 & 0.5351800 \\
0.5 & 0.1101025 & 0.2026902 & 0.2777854 & 0.3378061 & 0.3858145 & 0.4245707 & 0.4562607 & 0.4825283 \\
0.6 & 0.1021902 & 0.1834874 & 0.2471040 & 0.2969560 & 0.3364634 & 0.3682520 & 0.3942401 & 0.4158104 \\
\hline
\end{tabular}

Table 10 Values of $f_{0}^{2}$ for the case $\lambda=0.2, \beta v^{2}=0.25$

\begin{tabular}{lllllllll}
\hline$\eta_{0} \downarrow / \theta v^{2} \rightarrow$ & 0.25 & 0.50 & 0.75 & 1.00 & 1.25 & 1.50 & 1.75 & 2.00 \\
\hline 0.1 & 0.2200395 & 0.3539169 & 0.4451356 & 0.5118180 & 0.5629637 & 0.6035890 & 0.6367285 & 0.6643341 \\
0.2 & 0.2125212 & 0.3421194 & 0.4305462 & 0.4952475 & 0.5449058 & 0.5843679 & 0.6165695 & 0.6434008 \\
0.3 & 0.2000783 & 0.3225674 & 0.4063440 & 0.4677398 & 0.5149130 & 0.5524300 & 0.5830621 & 0.6085969 \\
0.4 & 0.1828486 & 0.2954343 & 0.3727072 & 0.4294674 & 0.4731484 & 0.5079279 & 0.5363488 & 0.5600553 \\
0.5 & 0.1610389 & 0.2609806 & 0.3299035 & 0.3806889 & 0.4198565 & 0.4510904 & 0.4766430 & 0.4979747 \\
0.6 & 0.1349428 & 0.2195764 & 0.2783132 & 0.3217720 & 0.3553841 & 0.3822418 & 0.4042464 & 0.4226362 \\
\hline
\end{tabular}

Table 11 Values of $f_{0}^{2}$ for the case $\lambda=0.2, \beta v^{2}=1.75$

\begin{tabular}{lllllllll}
\hline$\eta_{0} \downarrow / \theta v^{2} \rightarrow$ & 0.25 & 0.50 & 0.75 & 1.00 & 1.25 & 1.50 & 1.75 & 2.00 \\
\hline 0.1 & 0.2448837 & 0.3915553 & 0.4890137 & 0.5584838 & 0.6105600 & 0.6510941 & 0.6835752 & 0.7102112 \\
0.2 & 0.2374215 & 0.3795154 & 0.4739330 & 0.5412533 & 0.5917339 & 0.6310380 & 0.6625417 & 0.6883821 \\
0.3 & 0.2249399 & 0.3594183 & 0.4487860 & 0.5125366 & 0.5603667 & 0.5976261 & 0.6275043 & 0.6520206 \\
0.4 & 0.2073859 & 0.3312331 & 0.4135669 & 0.4723459 & 0.5164819 & 0.5508890 & 0.5784973 & 0.6011628 \\
0.5 & 0.1847135 & 0.2949476 & 0.3682952 & 0.4207213 & 0.4601320 & 0.4908856 & 0.5155825 & 0.5358716 \\
0.6 & 0.1569152 & 0.2506022 & 0.3130459 & 0.3577574 & 0.3914206 & 0.4177226 & 0.4388662 & 0.4562506 \\
\hline
\end{tabular}

4.3 Field profiles of the semilocal noncommutative vortices

Finally, by applying the inverse Weyl transform we can find the profiles of the semilocal vortices for different values of the parameters. The formulas are

$$
\begin{aligned}
& \phi^{+}(x) * \bar{\phi}^{+}(x)=v^{2} \sum_{k=0}^{\infty} f_{k}^{2} f_{k, k}(x), \\
& \phi^{0}(x) * \bar{\phi}^{0}(x)=v^{2} \sum_{k=0}^{\infty} \eta_{k}^{2} f_{k, k}(x),
\end{aligned}
$$


Table 12 Values of $f_{0}^{2}$ for the case $\lambda=0.5, \beta v^{2}=0.25$

\begin{tabular}{lllllllll}
\hline$\eta_{0} \downarrow / \theta v^{2} \rightarrow$ & 0.25 & 0.50 & 0.75 & 1.00 & 1.25 & 1.50 & 1.75 & 2.00 \\
\hline 0.1 & 0.1602409 & 0.2737020 & 0.3585048 & 0.4244411 & 0.4772755 & 0.5206269 & 0.5568846 & 0.5876912 \\
0.2 & 0.1550665 & 0.2649169 & 0.3470589 & 0.4109533 & 0.4621707 & 0.5042094 & 0.5393793 & 0.5692690 \\
0.3 & 0.1464564 & 0.2503017 & 0.3280186 & 0.3885166 & 0.4370436 & 0.4768968 & 0.5102548 & 0.5386168 \\
0.4 & 0.1344382 & 0.2299062 & 0.3014492 & 0.3572060 & 0.4019748 & 0.4387729 & 0.4695963 & 0.4958196 \\
0.5 & 0.1190664 & 0.2038210 & 0.2674641 & 0.3171484 & 0.3570982 & 0.3899747 & 0.4175409 & 0.4410131 \\
0.6 & 0.1004407 & 0.1722034 & 0.2262524 & 0.2685494 & 0.3026267 & 0.3307163 & 0.3543005 & 0.3744046 \\
\hline
\end{tabular}

Table 13 Values of $f_{0}^{2}$ for the case $\lambda=0.5, \beta v^{2}=1.75$

\begin{tabular}{lllllllll}
\hline$\eta_{0} \downarrow / \theta v^{2} \rightarrow$ & 0.25 & 0.50 & 0.75 & 1.00 & 1.25 & 1.50 & 1.75 & 2.00 \\
\hline 0.1 & 0.2283353 & 0.3828428 & 0.4892865 & 0.5653020 & 0.6216879 & 0.6649564 & 0.6991253 & 0.7267608 \\
0.2 & 0.2233610 & 0.3731310 & 0.4759154 & 0.5492336 & 0.6036180 & 0.6453673 & 0.6783533 & 0.7050458 \\
0.3 & 0.2146406 & 0.3564993 & 0.4532769 & 0.5221908 & 0.5733087 & 0.6125753 & 0.6436251 & 0.6687711 \\
0.4 & 0.2015959 & 0.3323599 & 0.4209074 & 0.4838293 & 0.5305060 & 0.5663915 & 0.5947978 & 0.6178272 \\
0.5 & 0.1835371 & 0.3000318 & 0.3782747 & 0.4337543 & 0.4749187 & 0.5065990 & 0.5317079 & 0.5520893 \\
0.6 & 0.1597608 & 0.2588504 & 0.3248680 & 0.3715902 & 0.4062713 & 0.4329942 & 0.4542035 & 0.4714428 \\
\hline
\end{tabular}

Table 14 Values of $f_{0}^{2}$ for the case $\lambda=0.8, \beta v^{2}=0.25$

\begin{tabular}{lllllllll}
\hline$\eta_{0} \downarrow / \theta v^{2} \rightarrow$ & 0.25 & 0.50 & 0.75 & 1.00 & 1.25 & 1.50 & 1.75 & 2.00 \\
\hline 0.1 & 0.0867972 & 0.1612771 & 0.2255448 & 0.2813263 & 0.3300317 & 0.3728119 & 0.4106060 & 0.4441825 \\
0.2 & 0.0847113 & 0.1572219 & 0.2196691 & 0.2737899 & 0.3209924 & 0.3624182 & 0.3989940 & 0.4314747 \\
0.3 & 0.0811168 & 0.1502752 & 0.2096520 & 0.2609908 & 0.3056882 & 0.3448651 & 0.3794237 & 0.4100937 \\
0.4 & 0.0758505 & 0.1401799 & 0.1951888 & 0.2426072 & 0.2837994 & 0.3198458 & 0.3516071 & 0.3797730 \\
0.5 & 0.0687112 & 0.1266240 & 0.1759151 & 0.2182588 & 0.2549512 & 0.2870036 & 0.3152118 & 0.3402070 \\
0.6 & 0.0594862 & 0.1092859 & 0.1514638 & 0.1875696 & 0.2187798 & 0.2459975 & 0.2699244 & 0.2911117 \\
\hline
\end{tabular}

Table 15 Values of $f_{0}^{2}$ for the case $\lambda=0.8, \beta v^{2}=1.75$

\begin{tabular}{lllllllll}
\hline$\eta_{0} \downarrow / \theta v^{2} \rightarrow$ & 0.25 & 0.50 & 0.75 & 1.00 & 1.25 & 1.50 & 1.75 & 2.00 \\
\hline 0.1 & 0.2077608 & 0.3713083 & 0.4891521 & 0.5729292 & 0.6338726 & 0.6796519 & 0.7151113 & 0.7433168 \\
0.2 & 0.2061466 & 0.3647761 & 0.4778972 & 0.5581057 & 0.6164778 & 0.6603812 & 0.6944332 & 0.7215522 \\
0.3 & 0.2023295 & 0.3526599 & 0.4582084 & 0.5327548 & 0.5870391 & 0.6279448 & 0.6597363 & 0.6851018 \\
0.4 & 0.1948819 & 0.3334512 & 0.4289408 & 0.4960701 & 0.5449875 & 0.5819318 & 0.6107156 & 0.6337338 \\
0.5 & 0.1822157 & 0.3055486 & 0.3888778 & 0.4471780 & 0.4896931 & 0.5218790 & 0.5470224 & 0.5671795 \\
0.6 & 0.1628170 & 0.2675205 & 0.3369334 & 0.3852825 & 0.4205688 & 0.4473456 & 0.4683193 & 0.4851761 \\
\hline
\end{tabular}

$$
\begin{aligned}
& B(x)=v^{2} \sum_{k=0}^{\infty}\left(1-\lambda+\lambda \beta v^{2}\left(f_{k}^{2}+\eta_{k}^{2}\right)\right) \\
& \times\left(1-f_{k}^{2}-\eta_{k}^{2}\right) f_{k, k}(x)
\end{aligned}
$$

We illustrate the results for several cases in Figs. 7, 8, 9, 10, 11 and 12 , where $\phi^{+}(x) * \bar{\phi}^{+}(x), \phi^{0}(x) * \bar{\phi}^{0}(x)$ and $B(x)$ are plotted, respectively, in red, blue, and green. In these examples we have chosen an intermediate value for $\eta_{0}$ and 
Table 16 Values of $g_{0}^{2}$ for commutative semilocal vortices

\begin{tabular}{lllllll}
\hline Parameter values $\downarrow$ & \multicolumn{7}{l}{$\eta 0$} & & & \\
\cline { 2 - 6 } & 0.1 & 0.2 & 0.3 & 0.4 & 0.5 & 0.6 \\
\hline$\lambda=0$ & 0.7191 & 0.6928 & 0.6494 & 0.5898 & 0.5151 & 0.4269 \\
$\lambda=0.2, \beta v^{2}=0.25$ & 0.5863 & 0.5655 & 0.5311 & 0.4836 & 0.4238 & 0.3527 \\
$\lambda=0.2, \beta v^{2}=1.75$ & 0.6522 & 0.6328 & 0.6002 & 0.5541 & 0.4942 & 0.4201 \\
$\lambda=0.8, \beta v^{2}=0.25$ & 0.1875 & 0.1833 & 0.1759 & 0.1650 & 0.1500 & 0.1304 \\
$\lambda=0.8, \beta v^{2}=1.75$ & 0.4444 & 0.4478 & 0.4501 & 0.4469 & 0.4327 & 0.4015 \\
$\lambda=1, \beta v^{2}=0.25$ & 0.0531 & 0.0548 & 0.0570 & 0.0587 & 0.0590 & 0.0566 \\
$\lambda=1, \beta v^{2}=1.75$ & 0.3717 & 0.3838 & 0.3991 & 0.4111 & 0.4126 & 0.3958 \\
\hline
\end{tabular}

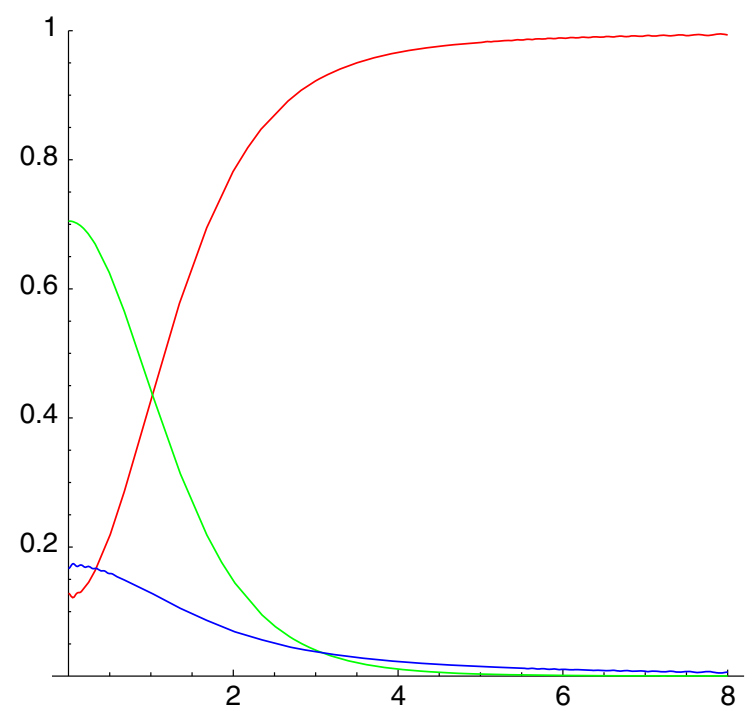

Fig. 7 The cases $\lambda=0, \eta_{0}=0.4, \theta v^{2}=0.25$

three values for the interpolating parameter, corresponding to semilocal Nielsen-Olesen vortices, semilocal Chern-Simons vortices, and a third case just in the middle of the range of $\lambda$. We have fixed the $\beta$ parameter to a value $\beta v^{2}=1$ and present, for each value of $\lambda$, solutions for both small $\left(\theta v^{2}=0.25\right)$ and large $\left(\theta v^{2}=1.75\right)$ noncommutativities. Some features that we can appreciate looking at the figures are the following:

- In the case of small $\theta v^{2}$ the profile of the magnetic field has a maximum at the center of the Nielsen-Olesen vortex, but it is ring-shaped for the Chern-Simons case; for the intermediate $\lambda=0.5$ solution the maximum is still there, although flatter.

- This pattern changes when the noncommutativity is large. In this case, the magnetic field profiles for $\lambda=0$ and $\lambda=0.5$ are nearly the same and, although with a slightly flatter maximum, the magnetic field remains concentrated at the core of the vortex also for $\lambda=1$.

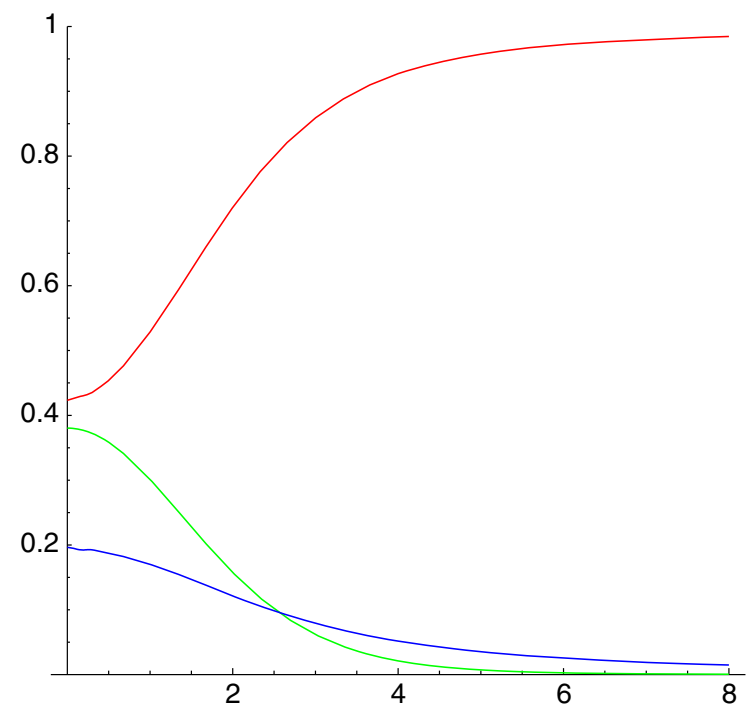

Fig. 8 The cases $\lambda=0, \eta_{0}=0.4, \theta v^{2}=1.75$

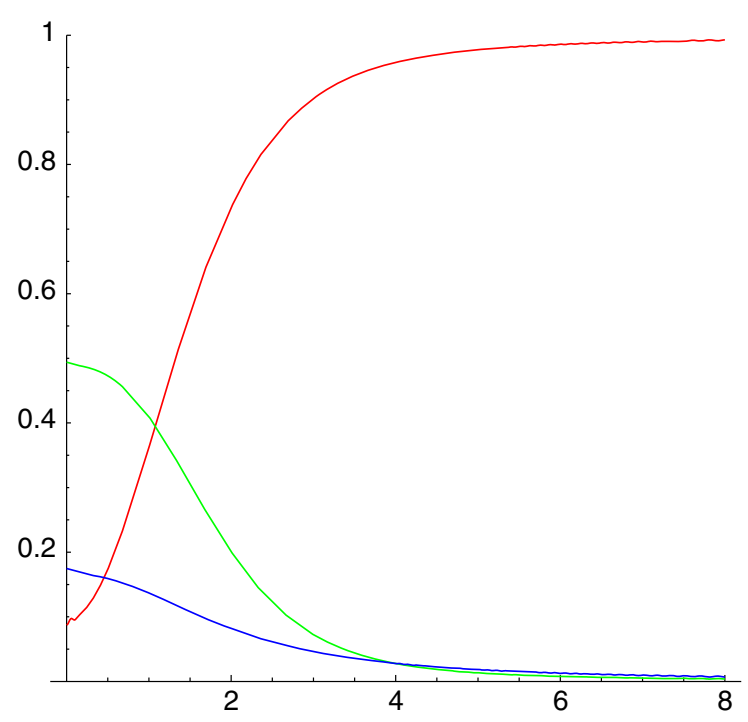

Fig. 9 The cases $\lambda=0.5, \eta_{0}=0.4, \theta v^{2}=0.25, \beta v^{2}=1$ 


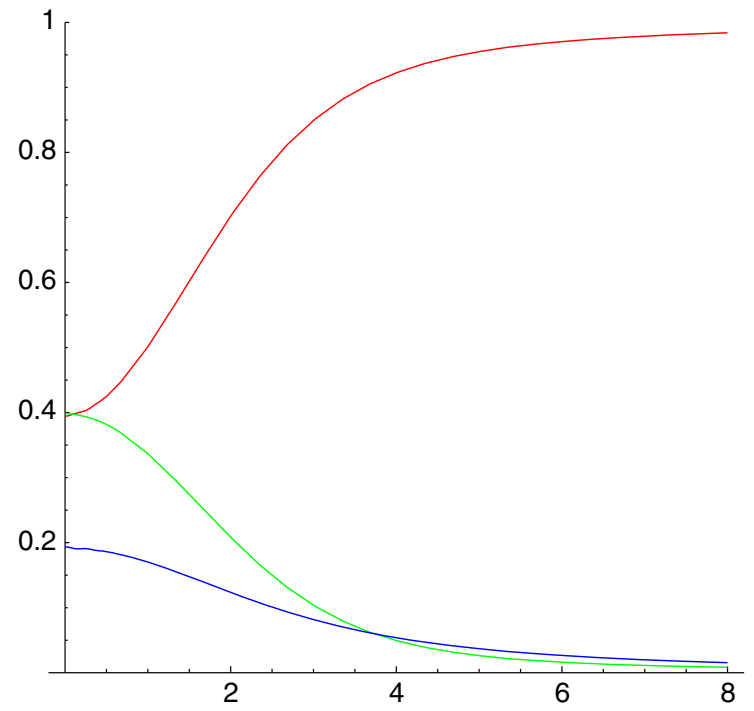

Fig. 10 The cases $\lambda=0.5, \eta_{0}=0.4, \theta v^{2}=1.75, \beta v^{2}=1$

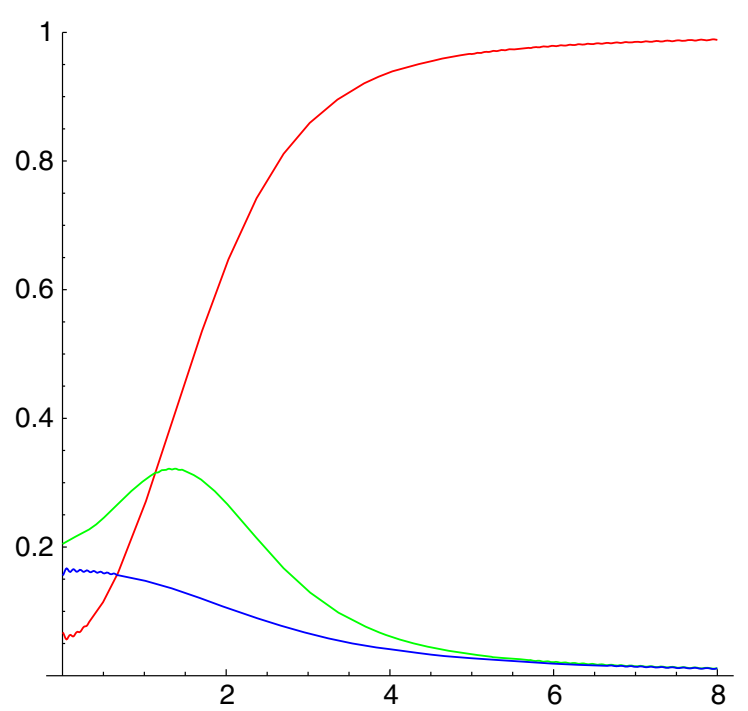

Fig. 11 The cases $\lambda=1, \eta_{0}=0.4, \theta v^{2}=0.25, \beta v^{2}=1$

- The magnitude of the upper component of the scalar field is minimum at the origin. The value of $\phi^{+}(0) * \bar{\phi}^{+}(0)$ decreases with $\lambda$, both for small and large $\theta$.

- For the three values of $\lambda, \phi^{+}(0) * \bar{\phi}^{+}(0)$ is higher for larger noncommutativity. Accordingly, the growth with distance of $\phi^{+}(x) * \bar{\phi}^{+}(x)$ is less steep in that case.

- The magnitude of the lower component of the scalar field has a maximum at the vortex core. There is, for the three values of $\lambda$, some increase of the value of $\phi^{0}(0) * \bar{\phi}^{0}(0)$ when $\theta v^{2}=1.75$ as compared with $\theta v^{2}=0.25$, but the effect is small. In all cases, $\phi^{0}(x) * \bar{\phi}^{0}(x)$ converges quite slowly to its asymptotic value, and the higher the noncommutativity, the slower the convergence.

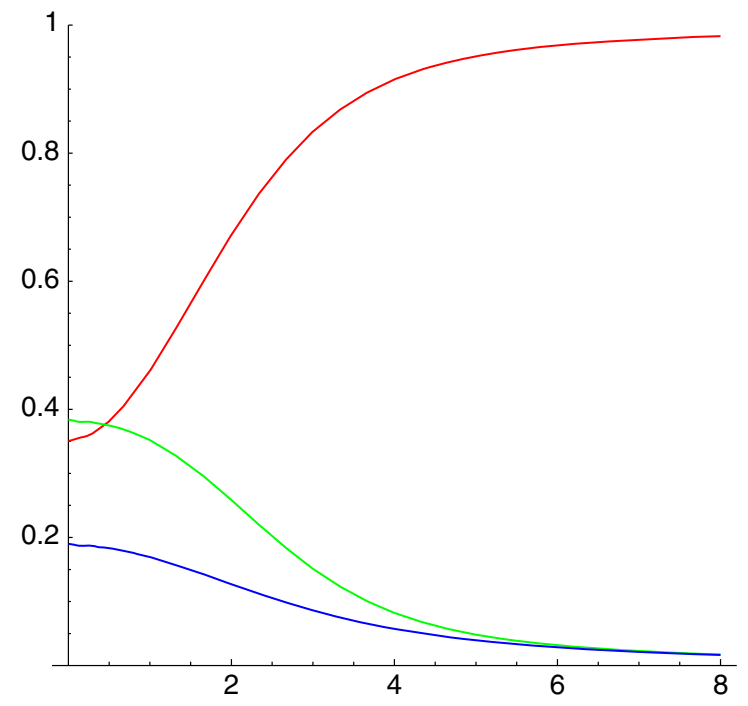

Fig. 12 The cases $\lambda=1, \eta_{0}=0.4, \theta v^{2}=1.75, \beta v^{2}=1$

\section{Conclusions and outlook}

In this paper we have studied the standard and semilocal noncommutative generalized AHM with dielectric function, showing that they admit a Bogomolny splitting and have, therefore, stable vorticial solutions whose energy is proportional to the magnetic flux. By changing the dielectric function it is possible to model the vortices in a variety of shapes. For the $U(1)$ model, we have focused in the case of unit vorticity and provided a number of solutions with different values of the non-dimensional parameters $\theta v^{2}$ and $\beta v^{2}$, finding vorticial profiles which interpolate between those of the noncommutative Nielsen-Olesen and Chern-SimonsHiggs cases. We have checked numerically that, for the case of $\theta \rightarrow 0$, regular solutions exist which converge to the vortices of the commutative model. The noncommutative $S U(2) \times U(1)$ semilocal model with dielectric function has also been investigated along the same lines, and their selfduality equations have been solved numerically for a variety of values of the above mentioned parameters and also of the coefficient $\eta_{0}$ which measures the degree of departure between standard and semilocal vortices.

Finally, let us make a couple of comments on some possible directions to extend this work in future research. Here we have concentrated in the case of a single vortex but, as is well known [24], the commutative self-duality equations admit multivortex solutions spanning a moduli space which has dimension $2 n$ in the topological sector of winding number $n$ [25]. It would be interesting to elaborate on the generalization of this result to the noncommutative cases with dielectric function that we have been studying. For the $U(1)$ case, for instance, if we shift the scalar and gauge fields of a vortex under the condition that the self-duality equations continue to be satisfied to linear order in the deformations $\delta \phi, \delta A_{k}$, 
we arrive at an equation of the form $\mathcal{D} \Psi=0$ where

$\mathcal{D}=\left(\begin{array}{llll}D_{1}+i D_{2} & 0 & -i R_{\phi} & R_{\phi} \\ 0 & \bar{D}_{1}+i \bar{D}_{2} & i L_{\bar{\phi}} & L_{\bar{\phi}} \\ \nabla_{2} & -\nabla_{1} & U_{R_{\bar{\phi}}} & U_{L_{\phi}} \\ \nabla_{1} & \nabla_{2} & i R_{\bar{\phi}} & -i L_{\phi}\end{array}\right)$

and

$\Psi=\left(\begin{array}{l}\delta \phi \\ \delta \bar{\phi} \\ \delta A_{1} \\ \delta A_{2}\end{array}\right)$

The first three rows in $\mathcal{D}$ come form the linearization of (6)(7), whereas the fourth one is a background gauge condition suitable to remove the spurious deformations which amount only to a change of gauge. The elements of $\mathcal{D}$ are operators whose action on the deformations is as follows:

$$
\begin{aligned}
& D_{k} \delta X=\partial_{k} \delta X-i A_{k} * \delta X \\
& \bar{D}_{k} \delta X=\partial_{k} \delta X+i \delta X * A_{k} \\
& \nabla_{k} \delta X=\partial_{k} \delta X-i A_{k} * \delta X+i \delta X * A_{k} \\
& R_{Y} \delta X=\delta X * Y \quad L_{Y} \delta X=Y * \delta X \\
& U_{K_{Y}} \delta X=\lambda \beta\left(K_{Y} \delta X\right) *\left(v^{2}-\phi * \bar{\phi}\right) \\
& -[(1-\lambda)+\lambda \beta \phi * \bar{\phi}] *\left(K_{Y} \delta X\right) .
\end{aligned}
$$

In the commutative case, the dimension of the vortex moduli space $\mathcal{M}$ is given by the index of $\mathcal{D}$ [25]

$\operatorname{dim} \mathcal{M}=\operatorname{ind} \mathcal{D}=\operatorname{dim} \operatorname{ker} \mathcal{D}-\operatorname{dim} \operatorname{ker} \mathcal{D}^{\dagger}$

and, given that this is a topological quantity, and the noncommutative self-duality equations are continuous deformations in the $\theta$ parameter of the commutative ones, we expect on general grounds that the result valid for commutative vortices is still valid for any $\theta$. Nevertheless, all the details of the computation, such as to establish a vanishing theorem for the kernel of $\mathcal{D}^{\dagger}$ or to evaluate the heat-kernel traces of the superpartner Laplacians $\mathcal{D}^{\dagger} \mathcal{D}$ and $\mathcal{D} \mathcal{D}^{\dagger}$, seem to be quite intricate for objects involving the Groenewold-Moyal product; see [26,27]. In particular, the coefficients of the asymptotic expansions of these heat-kernel traces split into three terms:

$a_{n}(\mathcal{O})=a_{n}^{L}(\mathcal{O})+a_{n}^{R}(\mathcal{O})+a_{n}^{\operatorname{mix}}(\mathcal{O})$

for $\mathcal{O}=\mathcal{D}^{\dagger} \mathcal{D}$ or $\mathcal{O}=\mathcal{D} \mathcal{D}^{\dagger}$, where $a_{n}^{L}(\mathcal{O})$ involves only the fields entering in $\mathcal{O}$ as left Moyal multipliers, $a_{n}^{R}(\mathcal{O})$ includes only right Moyal multipliers, and $a_{n}^{\operatorname{mix}}(\mathcal{O})$ is given by a combination of fields of both types. Furthermore, this last term is divergent as $\theta^{-1}$ when the commutativity of the plane is restored. Thus, an issue to be clarified is if the good behavior in the limit $\theta \rightarrow 0$ of the solutions reported here is enough to ensure that the coefficients $a_{n}^{\text {mix }}$ coming from the deformation operator $\mathcal{D}$ effectively vanish. On the other hand, the heat-kernel coefficients are interesting also from the point of view of computing the quantum corrections to the semiclassical energy of vortices. In fact, the main part of this correction comes from the trace of $\mathcal{D}^{\dagger} \mathcal{D}$ once a convenient regularization scheme, based for instance on zeta-function methods [21], is stipulated. For the commutative $U(1)$ and semilocal vortices, the computation of the leading $a_{n}\left(\mathcal{D}^{\dagger} \mathcal{D}\right)$ needeed for the quantum corrections has been performed in $[28,29]$. We think that it would be a worthwhile project to study the precise way in which the methods described there can be generalized in order to be applied to the case of noncommutative solutions.

Open Access This article is distributed under the terms of the Creative Commons Attribution License which permits any use, distribution, and reproduction in any medium, provided the original author(s) and the source are credited.

Funded by $\mathrm{SCOAP}^{3}$ / License Version CC BY 4.0.

\section{References}

1. M.R. Douglas, N.A. Nekrasov, Rev. Mod. Phys. 73, 977 (2001)

2. R.J. Szabo, Phys. Rep. 378, 207 (2003)

3. R. Jackiw, Nucl Phys B Proc Suppl 127, 53-62 (2004). hep-th/0305027

4. T.C. Adorno, D.M. Gitman, A.E. Shabad, D.V. Vassilevich, Phys. Rev. D 84, 085031 (2011)

5. J.A Harvey, Komaba Lectures on Noncommutative Solitons and D-Branes. hep-th/0102076

6. J. Lee, S. Nam, Phys. Lett. B 261, 437 (1991)

7. D. Bazeia, Phys. Rev. D 46, 1879 (1992)

8. W. García Fuertes, J. Mateos Guilarte, Eur. Phys. J. C 9, 535 (1999)

9. W. García Fuertes, J. Mateos Guilarte, Eur. Phys. J. C 9, 167 (1999)

10. W. García Fuertes, J. Mateos Guilarte. J. Math. Phys. 38, 6214 (1996)

11. D. Bazeia, E. da Hora, C. dos Santos, R. Menezes, Eur. Phys. J. C 71, 1833 (2011)

12. D. Bazeia, R. Casana, E. da Hora, R. Menezes, Phys. Rev. D 85, 125028 (2012)

13. G.S. Lozano, E.F. Moreno, F.A. Schaposnik, Phys. Lett. B 504, $117(2001)$

14. G.S. Lozano, E.F. Moreno, F.A. Schaposnik, JHEP 0102, 036 (2001)

15. F. Schaposnik, Three Lectures on Noncommutative Field Theories. hep-th/0408132

16. L. Álvarez-Gaumé, S.R. Wadia, Phys. Lett. B 501, 319 (2001)

17. D.P. Jatkar, G. Mandal, S.R. Wadia, JHEP 0009, 018 (2000)

18. E. Witten, Noncommutative Tachyons and String Field Theory. hep-th/0006071

19. T. Vachaspati, A. Achúcarro, Phys. Rev. D 44, 3067 (1991)

20. G.W. Gibbons, M.E. Ortiz, F. Ruiz, T.M. Samols, Nucl. Phys. B 385, 127 (1992)

21. J. Mateos Guilarte, A. Alonso Izquierdo, W. García Fuertes, M. de la Torre Mayado, M.J. Senosiain, in Proceedings of the 5th International School on Field Theory and Gravitation (Cuiabá, Brazil, PoS (ISFTG), 2009)

22. M. Hindmarsh, Phys. Rev. Lett. 68, 1263 (1992)

23. W. García Fuertes, J. Mateos Guilarte. J. Math. Phys. 37, 554 (1996)

24. A. Jaffe, C. Taubes, Vortices and Monopoles, Structure of Static Gauge Theories (Birkhäuser, Basel, 1980)

25. E.J. Weinberg, Phys. Rev. D 19, 3008 (1979) 
26. D.V. Vassilevich, SIGMA 3, 093 (2007). hep-th/07084209

27. R.A. Konoplya, D.V. Vassilevich, JHEP 0801, 068 (2008)

28. A. Alonso Izquierdo, W. García Fuertes, J. Mateos Guilarte, M. de la Torre Mayado, Phys. Rev. D 71, 125010 (2005)
29. A. Alonso Izquierdo, W. García Fuertes, J. Mateos Guilarte, M. de la Torre Mayado, Nucl. Phys. B 797, 431463 (2008) 